\title{
General dentists can provide successful implant overdentures with minimal training
}

\author{
Can inexperienced dentists provide two-implant overdentures that are as \\ satisfactory, and at the same cost, as those provided by experienced prosthodontists?
}

\author{
Esfandiari S, Lund JP, Thomason JM, et al. Can general dentists \\ produce successful implant overdentures with minimal training? \\ J Dent 2006; 34:796-801
}

Design This randomised controlled trial (RCT) used a $2 \times 2$ factorial design.

Intervention Edentulous people of age $>65$ years were enrolled in the $\mathrm{RCT}$, which was designed to compare the effects on nutrition of conventional and two-implant mandibular overdentures. They were randomly assigned to groups treated either by an experienced prosthodontist or by a newly-graduated dentist who had undergone minimal training in implant treatment.

Outcome measure The change in patient ratings of satisfaction after treatment, laboratory costs, and the number of unscheduled visits up to 6 months following delivery of the prosthesis were compared. To determine the clinicians' perception of difficulty in providing the two prosthetic treatments, a short questionnaire was administered using telephone interviews.

Results Data were gathered from the first 140 patients who had been treated by either one of the three prosthodontists or by one of the eight newly graduated dentists. The prosthodontists provided a total of 28 implant overdentures and 46 conventional dentures, whereas the inexperienced dentists provided 33 prostheses for each kind. Satisfaction was significantly higher with implant overdentures than with conventional dentures, but there were no differences in scores for either prosthesis between the groups treated by experienced specialists or new dentists. The laboratory costs of fabricating implant overdentures were significantly higher than the cost of conventional dentures. There was no significant difference between the two groups of clinicians in mean laboratory costs, however, for either conventional dentures or implant overdentures. There was no significant between-group difference in the number of unscheduled visits for either prosthesis. Furthermore, six of the seven inexperienced dentists reported that they found the mandibular two-implant overdenture easier to provide than the conventional denture.

Conclusions Inexperienced dental practitioners can provide successful mandibular two-implant overdentures for their patients with minimal training.

\section{Commentary}

There is much evidence to show that osseointegrated implants are reliable in their success and that implant-supported overdentures can provide significant benefits for edentulous patients. ${ }^{1,2}$ This timely study addresses a current area of contention. How much training do dentists need to provide implant-supported prostheses?

The data in this well-constructed RCT were extracted from a large longitudinal study which is investigating the effect of the provision of implant-supported overdentures on nutritional status. The authors have taken the opportunity to compare outcomes within the trial of treatment provided by newly graduated dentists and by prosthodontists.

All the prosthodontists had experience of complete dentures and implant treatment. The newly graduated dentists were on a postgraduate hospital training programme. It is important to note that these newly graduated dentists carried out treatment under supervision. It is not entirely clear how much advice and support was provided but it would have been more than was available, for example, to most dentists in general practice.

Outcome measures used were patient satisfaction, cost and number of additional visits/ appointments. Patient satisfaction is perhaps the most important of these but is most difficult to measure. The authors chose to determine satisfaction by means of a visual analogue scale response to what appears to have been a single question. All but one of the newly graduated dentists found the overdenture treatment to be easier to provide than the conventional denture treatment.

The study certainly supports the contention that the prosthetic phase of provision of implant-supported prostheses is relatively straightforward. Suitably experienced educators may be encouraged to provide training opportunities for dentists who wish to offer this form of treatment in their practice. Although the answer to the question posed by the authors, "Can general dentists produce successful implant overdentures with minimal training?" is undoubtedly yes, exactly what constitutes minimal training remains to be answered.

\section{Practice point}

General dentists can produce implant overdentures but the minimum level of training required to do that remains to be determined.

\section{Rob Jagger}

Division of Restorative Dentistry, Department of Oral and Dental Science, University of Bristol, Bristol, UK

1. Raghoebar GM, Meijer HJ, Van't Hof M, Stegenga B, Vissink A. A randomized prospective clinical trial on the effectiveness of three treatment modalities for patients with lower denture problems. A 10 year follow-up study on patient satisfaction. Int J Oral Maxillofac Surg 2003; 32:498-503.

2. Thomason JM, Lund JP, Chehade A, Feine JS. Patient satisfaction with mandibular implant overdentures and conventional dentures 6 months after delivery. Int J Prosthodont 2003; 16:467-473.

Evidence-Based Dentistry (2007) 8, 5-6. doi:10.1038/sj.ebd.6400496
Address for correspondence: JS Feine, Faculty of Dentistry,

McGill University, 3640 University Street, Montreal, Quebec, Canada H3A 2B2.

E-mail: joicelyne.feine@mcgill.ca 